\title{
MAXIMUM ORDERS OF CYCLIC AND ABELIAN EXTENDABLE ACTIONS ON SURFACES
}

\author{
CHAO WANG AND YIMU ZHANG
}

\begin{abstract}
A faithful action of a group $G$ on the genus $g>1$ orientable closed surface $\Sigma_{g}$ is extendable (over the three dimensional sphere $S^{3}$ ), with respect to an embedding $e: \Sigma_{g} \hookrightarrow S^{3}$, if $G$ can act on $S^{3}$ such that $h \circ e=e \circ h$ for any $h \in G$. We show that the maximum order of extendable cyclic group actions on $\Sigma_{g}$ is $4 g+4$ when $g$ is even, and is $4 g-4$ when $g$ is odd; the maximum order of extendable abelian group actions on $\Sigma_{g}$ is $4 g+4$. We also give the maximum orders of cyclic and abelian group actions on handlebodies.
\end{abstract}

\section{INTRODUCTION}

Let $\Sigma_{g}$ be the genus $g>1$ orientable closed surface, $S^{3}$ be the three dimensional sphere, and $G$ be a finite group.

Definition 1.1. A faithful $G$-action on $\Sigma_{g}$ is extendable, with respect to an embedding $e: \Sigma_{g} \hookrightarrow S^{3}$, if $G$ can act on $S^{3}$ such that $h \circ e=e \circ h$ for any $h \in G$. We also say that such an action on $\Sigma_{g}$ is extendable over $S^{3}$.

In this paper we consider the following question in the smooth category (namely all manifolds and maps are smooth): what is the maximum order of extendable cyclic (abelian) group actions on $\Sigma_{g}$ ? And following is our main result.

Theorem 1.2. (1). The maximum order of extendable cyclic group actions on $\Sigma_{g}$ is $4 g+4$ when $g$ is even, and is $4 g-4$ when $g$ is odd.

(2). The maximum order of extendable abelian group actions on $\Sigma_{g}$ is $4 g+4$.

Let $V_{g}$ be the genus $g>1$ orientable handlebody. The question below is closely related to the above one: what is the maximum order of cyclic (abelian) group actions on $V_{g}$ ? And we have the following answer to it.

Theorem 1.3. (1). The maximum order of cyclic group actions on $V_{g}$ is

$$
\operatorname{Max}\left\{2[m, n] \mid g=[m, n]-\frac{m+n}{(m, n)}+1, m, n \in \mathbb{Z}_{+}, 2 \nmid m, 2 \nmid n\right\}
$$

when $g$ is even, and is

$$
\operatorname{Max}\left\{2 k n \mid g=k n-k+1, k, n \in \mathbb{Z}_{+}, 2 \nmid k, 2 \nmid n\right\}
$$

when $g$ is odd.

(2). The maximum order of abelian group actions on $V_{g}$ is $4 g+4$ when $g \neq 5$, and is 32 when $g=5$. It is equal to the maximum order of abelian group actions on $\Sigma_{g}$.

2010 Mathematics Subject Classification. Primary 57M60, 57S17, 57S25.

Key words and phrases. maximum order, extendable action, orbifold.

The authors were supported by National Natural Science Foundation of China (11371034).

The authors would like to thank Prof. Shicheng Wang and the referee for their helpful advices. 
Here $\mathbb{Z}_{+}$is the set of positive integers, $[m, n]$ is the lowest common multiple of $m$ and $n$, and $(m, n)$ is the greatest common divisor of $m$ and $n$.

If $g$ is even, then let $m=n=g+1$. And if $g$ is odd, then let $k=1, n=g$. Then by Theorem 1.3, it is easy to see that the maximum order of cyclic group actions on $V_{g}$ is at least $2 g+2$ when $g$ is even, and is at least $2 g$ when $g$ is odd.

Remark 1.4. (1). The extendable action in Definition 1.1 was firstly defined in [14]. And following notations were used in [14.

$C_{g}\left(A_{g}\right)$ : the maximum order of orientation-preserving cyclic (abelian) group actions on $\Sigma_{g}$.

$C H_{g}\left(A H_{g}\right)$ : the maximum order of orientation-preserving cyclic (abelian) group actions on $V_{g}$.

$C E_{g}\left(A E_{g}\right)$ : the maximum order of extendable cyclic (abelian) group actions on $\Sigma_{g}$, which preserve both the orientations of $\Sigma_{g}$ and $S^{3}$.

All these numbers have been determined, see [4, 11, 13, for $C_{g}$; 6] for $A_{g}$; 7] for $C H_{g}$; [7, 8, 14] for $A H_{g}$; 14] for $C E_{g}$ and $A E_{g}$. We summarize them in Table [1]

TABLE 1. Maximum orders in orientation-preserving case

\begin{tabular}{|l|l|l|l|}
\hline$C_{g}$ & $4 g+2$ & $A_{g}$ & $4 g+4$ \\
\hline$C H_{g}$ & $2 g+2(g$ even $), 2 g(g$ odd $)$ & $A H_{g}$ & $2 g+2(g \neq 5), 16(g=5)$ \\
\hline$C E_{g}$ & $2 g+2(g$ even $), 2 g-2(g$ odd $)$ & $A E_{g}$ & $2 g+2$ \\
\hline
\end{tabular}

(2). The maximum order of orientation-reversing periodic maps on $\Sigma_{g}$ is $4 g+4$ when $g$ is even, and is $4 g-4$ when $g$ is odd. Hence the maximum order of cyclic group actions on $\Sigma_{g}$ is $4 g+4$ when $g$ is even, and is $4 g+2$ when $g$ is odd, see [13].

By comparing Theorem 1.2 and 1.3 with Remark 1.4, we see that in most cases if the maximum order is achieved, then the group will contain an element reversing the orientation of either $\Sigma_{g}, V_{g}$ or $S^{3}$.

Definition 1.5. For each given $g>1$, define

$C_{g}^{-}$: the maximum order of cyclic group actions on $\Sigma_{g}$, which contains an element reversing the orientation of $\Sigma_{g}$.

$A_{g}^{-}$: the maximum order of abelian group actions on $\Sigma_{g}$, which contains an element reversing the orientation of $\Sigma_{g}$.

$\mathrm{CH}_{g}^{-}$: the maximum order of cyclic group actions on $V_{g}$, which contains an element reversing the orientation of $V_{g}$.

$A H_{g}^{-}$: the maximum order of abelian group actions on $V_{g}$, which contains an element reversing the orientation of $V_{g}$.

Definition 1.6. Define five types of extendable $G$-actions on $\Sigma_{g}$ as following:

$(+,+): G$ preserves both the orientations of $\Sigma_{g}$ and $S^{3}$.

$(+,-): G$ preserves the orientation of $\Sigma_{g}$, and there exists $h_{1} \in G$ such that $h_{1}$ reverses the orientation of $S^{3}$.

$(-,+): G$ preserves the orientation of $S^{3}$, and there exists $h_{2} \in G$ such that $h_{2}$ reverses the orientation of $\Sigma_{g}$.

$(-,-)$ : for any $h \in G$, either $h$ preserves both the orientations of $\Sigma_{g}$ and $S^{3}$, or $h$ reverses both the orientations of $\Sigma_{g}$ and $S^{3}$.

(Mix): there exists $h_{1} \in G$ such that $h_{1}$ preserves the orientation of $\Sigma_{g}$ and reverses the orientation of $S^{3}$, and there exists $h_{2} \in G$ such that $h_{2}$ reverses the orientation of $\Sigma_{g}$ and preserves the orientation of $S^{3}$. 
Definition 1.7. For a given type $\mathcal{T}$ in Definition 1.6 and a given $g>1$, define

$C E_{g} \mathcal{T}$ : the maximum order of type $\mathcal{T}$ extendable cyclic group actions on $\Sigma_{g}$, if such an action exists.

$A E_{g} \mathcal{T}$ : the maximum order of type $\mathcal{T}$ extendable abelian group actions on $\Sigma_{g}$, if such an action exists.

Clearly we have $C E_{g}(+,+)=C E_{g}$ and $A E_{g}(+,+)=A E_{g}$. For other types we have the following result. By Remark 1.4, it will give us Theorem 1.2.

Proposition 1.8. For each type $\mathcal{T} \neq(+,+)$ and genus $g>1, C E_{g} \mathcal{T}$ and $A E_{g} \mathcal{T}$ are given in Table 0 . The notation "-" means that the type $\mathcal{T}$ extendable action does not exist.

TABLE 2. Maximum orders of type $\mathcal{T}$ extendable actions

\begin{tabular}{|c|l|l|}
\hline $\mathcal{T}$ & $C E_{g} \mathcal{T}$ & $A E_{g} \mathcal{T}$ \\
\hline$(+,-)$ & $2 g+2$ & $4 g+4$ \\
\hline$(-,+)$ & $4 g+4$ (g even $), 4 g-4(g$ odd $)$ & $4 g+4$ \\
\hline$(-,-)$ & $2 g+2$ (g even $), 2 g(g$ odd $)$ & $4 g+4$ \\
\hline$($ Mix $)$ & - & $2 g+4$ (g even $),-(g$ odd $)$ \\
\hline
\end{tabular}

We see that $C E_{g}(-,+)=C_{g}^{-}$and $A E_{g}(+,-)=A_{g}$, see Remark 1.4. Namely some kinds of maximum symmetries on surfaces are extendable over $S^{3}$.

We also have the following result. By Remark 1.4, it will give us Theorem 1.3.

Proposition 1.9. For each genus $g>1, \mathrm{CH}_{g}^{-}$and $A H_{g}^{-}$are given in Table 3 , here $k, m, n \in \mathbb{Z}_{+}$. And $A_{g}^{-}$is equal to $A H_{g}^{-}$.

TABLE $3 . \mathrm{CH}_{g}^{-}$and $A H_{g}^{-}$

\begin{tabular}{|l|l|}
\hline$C H_{g}^{-}(g$ even $)$ & $\operatorname{Max}\{2[m, n] \mid g=[m, n]-(m+n) /(m, n)+1,2 \nmid m, 2 \nmid n\}$ \\
\hline$C H_{g}^{-}(g$ odd $)$ & $\operatorname{Max}\{2 k n \mid g=k n-k+1,2 \nmid k, 2 \nmid n\}$ \\
\hline$A H_{g}^{-}$ & $4 g+4(g \neq 5), 32(g=5)$ \\
\hline
\end{tabular}

In $\S 2$, we will give some examples which will give the lower bounds of maximum orders we have defined. In $\S 3$, we will give some known facts about group actions on manifolds, which will be used later. In $\S 4$, we will prove Proposition 1.9. Some results in $\S 4$ will be used in $\S 5$, where we will prove Proposition 1.8 .

\section{EXAMPLES}

In this section we will give three examples about extendable actions and one example of non-extendable action. The surfaces we constructed below may be nonsmooth, but using orbifold theory discussed in $\S 3$, they can be replaced by smooth ones. The following lemma is easy to verified.

Lemma 2.1. Let $\Sigma_{g}$ be a closed subsurface in $S^{3}$. Suppose $h$ is an automorphism of $S^{3}$ preserving $\Sigma_{g}$. If some of the following conditions (a), (b) and (c) happen, then exactly two of them happen.

(a). $h$ reveres the orientation of $S^{3}$.

(b). $h$ reveres the orientation of $\Sigma_{g}$.

(c). $h$ changes the two sides of $\Sigma_{g}$. 
Example 2.2 (Cage). Given $g>1$, we will construct some cyclic and abelian group actions on a Heegaard splitting of $S^{3}$.

Let $S^{3}$ be the unit sphere in $\mathbb{C}^{2}$ :

$$
S^{3}=\left\{\left.\left(z_{1}, z_{2}\right) \in \mathbb{C}^{2}|| z_{1}\right|^{2}+\left|z_{2}\right|^{2}=1\right\} .
$$

Let

$$
\begin{aligned}
a_{m} & =\left(e^{\frac{m \pi}{2} i}, 0\right), m=0,1,2,3, \\
b_{n} & =\left(0, e^{\frac{n \pi}{g+1} i}\right), n=0,1, \cdots, 2 g+1 .
\end{aligned}
$$

Connect each $a_{2 l}$ to each $b_{2 k}$ with the shortest geodesic in $S^{3}$, and connect each $a_{2 l+1}$ to each $b_{2 k+1}$ with the shortest geodesic in $S^{3}$, here $l=0,1$ and $k=0,1, \cdots, g$. Then we get two graphs $\Gamma_{g}^{c}, \Gamma_{g}^{c \prime} \in S^{3}$. $\Gamma_{g}^{c}$ contains $a_{2 l}, b_{2 k}$, and $\Gamma_{g}^{c \prime}$ contains $a_{2 l+1}$, $b_{2 k+1}$. Each graph has $g+3$ vertices and $2 g+2$ edges. They are in the dual positions as in Figure 1 $(g=2)$, here graphs have been projected into the three dimensional Euclidean space $E^{3}$ from $S^{3}-\{(-1,0)\}$. The vertex $a_{2}$ is at the infinity.

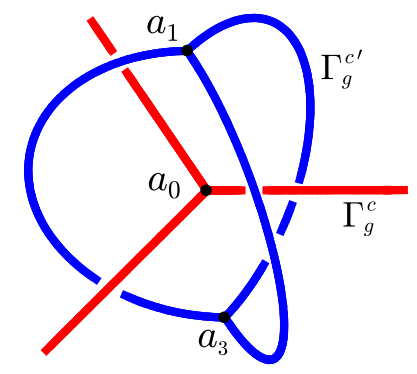

FiguRE 1. $\Gamma_{g}^{c}$ and $\Gamma_{g}^{c \prime}$ in $S^{3}$

Let $T$ be the following torus in $S^{3}$ :

$$
T=\left\{\left(z_{1}, z_{2}\right) \in S^{3}|| z_{1}|=| z_{2} \mid=\frac{\sqrt{2}}{2}\right\} .
$$

It splits $S^{3}$ into two solid tori $V_{1}$ and $V_{2}$ :

$$
\begin{aligned}
& V_{1}=\left\{\left(z_{1}, z_{2}\right) \in S^{3}|| z_{1} \mid \geq \frac{\sqrt{2}}{2}\right\}, \\
& V_{2}=\left\{\left(z_{1}, z_{2}\right) \in S^{3}|| z_{2} \mid \geq \frac{\sqrt{2}}{2}\right\} .
\end{aligned}
$$

Let $D_{1, m}$ and $D_{2, n}$ be following meridian disks in $V_{1}$ and $V_{2}$ respectively:

$$
\begin{aligned}
& D_{1, m}=\left\{\left(\sqrt{1-r^{2}} e^{\frac{\pi}{4} i+\frac{m \pi}{2} i}, r e^{\theta i}\right) \in S^{3} \mid 0 \leq r \leq \frac{\sqrt{2}}{2}, \theta \in \mathbb{R}\right\}, \\
& D_{2, n}=\left\{\left(r e^{\theta i}, \sqrt{1-r^{2}} e^{\frac{\pi}{2 g+2} i+\frac{n \pi}{g+1} i}\right) \in S^{3} \mid 0 \leq r \leq \frac{\sqrt{2}}{2}, \theta \in \mathbb{R}\right\} .
\end{aligned}
$$

Here $m=0,1,2,3$ and $n=0,1, \cdots, 2 g+1$. All the $D_{1, m}$ cut $V_{1}$ into 4 cylinders. And each cylinder contains exactly one $a_{m}$ for some $m$. All the $D_{2, n}$ cut $V_{2}$ into $2 g+2$ cylinders. And each cylinder contains exactly one $b_{n}$ for some $n$. Hence $T$ and all these disks cut $S^{3}$ into $2 g+6$ cylinders. Let $V_{g}^{c}$ be the union of cylinders intersecting $\Gamma_{g}^{c}$, and $V_{g}^{c^{\prime}}$ be the union of cylinders intersecting $\Gamma_{g}^{c^{\prime}}$. Then $V_{g}^{c}$ and $V_{g}^{c^{\prime}}$ are two handlebodies. $\partial V_{g}^{c}=\partial V_{g}^{c^{\prime}}$ is a Heegaard surface of $S^{3}$, and $\partial V_{g}^{c} \cong \Sigma_{g}$. 
Following three isometries on $S^{3}$ preserve the graph $\Gamma_{g}^{c} \cup \Gamma_{g}^{c^{\prime}}$, the torus $T$, and the union of the disks. They also preserve $\partial V_{g}^{c}$.

$$
\begin{aligned}
\tau_{g} & :\left(z_{1}, z_{2}\right) \mapsto\left(i z_{1}, e^{\frac{\pi}{g+1} i} z_{2}\right) \\
\rho:\left(z_{1}, z_{2}\right) & \mapsto\left(-z_{1}, z_{2}\right) \\
\sigma:\left(z_{1}, z_{2}\right) & \mapsto\left(\bar{z}_{1}, z_{2}\right)
\end{aligned}
$$

For even $g>1, \tau_{g}$ has order $4 g+4$ and $\tau_{g}^{2} \rho \sigma$ has order $2 g+2$.

For each $g>1, \tau_{g} \sigma$ has order $2 g+2$ and $\left\langle\tau_{g} \sigma, \rho\right\rangle,\left\langle\tau_{g}, \rho\right\rangle,\left\langle\tau_{g}^{2}, \rho, \sigma\right\rangle$ are all abelian groups of order $4 g+4$.

Notice that $\tau_{g}$ and $\rho$ preserve the orientation of $S^{3}$, and $\sigma$ reverses the orientation of $S^{3}$. Only $\tau_{g}$ changes the two sides of $\partial V_{g}^{c}$. Then combining with Lemma 2.1, we have extendable cyclic (abelian) group actions on $\Sigma_{g} \cong \partial V_{g}^{c}$ as following:

(1). For even $g>1,\left\langle\tau_{g}\right\rangle$ gives a type $(-,+)$ extendable cyclic group action of order $4 g+4$. This also realizes the maximum order of cyclic group actions on $\Sigma_{g}$ when $g$ is even.

(2). For even $g>1,\left\langle\tau_{g}^{2} \rho \sigma\right\rangle$ gives a type $(-,-)$ extendable cyclic group action of order $2 g+2$.

$\left(2^{\prime}\right)$. For odd $g>1$, by adding shortest geodesics $a_{0} a_{1}$ and $a_{0} a_{3}$ to $\Gamma_{g-1}^{c}{ }^{\prime}$, we get a graph $\Gamma_{g}$. The group $\left\langle\tau_{g-1}^{2} \rho \sigma\right\rangle$ preserves $\Gamma_{g}$. Hence it also preserves some regular neighbourhood $N\left(\Gamma_{g}\right)$ of $\Gamma_{g}$. Then $\left\langle\tau_{g-1}^{2} \rho \sigma\right\rangle$ gives a type $(-,-)$ extendable cyclic group action of order $2 g$ on $\Sigma_{g} \cong \partial N\left(\Gamma_{g}\right)$.

(3). For each $g>1,\left\langle\tau_{g} \sigma\right\rangle$ gives a type $(+,-)$ extendable cyclic group action of order $2 g+2$.

(4). For each $g>1,\left\langle\tau_{g} \sigma, \rho\right\rangle$ gives a type $(+,-)$ extendable abelian group action of order $4 g+4$. This also realizes the maximum order of orientation-preserving abelian group actions on $\Sigma_{g}$.

(5). For each $g>1,\left\langle\tau_{g}, \rho\right\rangle$ gives a type $(-,+)$ extendable abelian group action of order $4 g+4$.

(6). For each $g>1,\left\langle\tau_{g}^{2}, \rho, \sigma\right\rangle$ gives a type $(-,-)$ extendable abelian group action of order $4 g+4$.

Example 2.3 (Wheel). Given odd $g>1$, we will construct a cyclic group action on a Heegaard splitting of $S^{3}$.

Let $S^{3}$ and $T$ be in $\mathbb{C}^{2}$ as above. Let $L=L_{1} \cup L_{2}$ be the $(2,4)$-torus link in $T$ :

$$
\begin{aligned}
& L_{1}=\left\{\left(\frac{\sqrt{2}}{2} e^{2 \theta i}, \frac{\sqrt{2}}{2} e^{\theta i}\right) \in S^{3} \mid \theta \in \mathbb{R}\right\}, \\
& L_{2}=\left\{\left(\frac{\sqrt{2}}{2} e^{2 \theta i}, \frac{\sqrt{2}}{2} i e^{\theta i}\right) \in S^{3} \mid \theta \in \mathbb{R}\right\} .
\end{aligned}
$$

Let

$$
\begin{aligned}
a_{m} & =\left(\frac{\sqrt{2}}{2} e^{\frac{2 m \pi}{g-1} i}, \frac{\sqrt{2}}{2} e^{\frac{2 m \pi}{2 g-2} i}\right), m=0,1, \cdots, 2 g-3, \\
b_{n} & =\left(\frac{\sqrt{2}}{2} e^{\frac{(2 n+1) \pi}{g-1} i}, \frac{\sqrt{2}}{2} i e^{\frac{(2 n+1) \pi}{2 g-2} i}\right), n=0,1, \cdots, 2 g-3 .
\end{aligned}
$$

Then $a_{0}, a_{1}, \cdots, a_{2 g-3}$ are $2 g-2$ points in $L_{1}$, and $b_{0}, b_{1}, \cdots, b_{2 g-3}$ are $2 g-2$ points in $L_{2}$. Connect $a_{i}$ to $a_{i+g-1}$ with the shortest geodesic in $S^{3}$, and connect $b_{i}$ to $b_{i+g-1}$ with the shortest geodesic in $S^{3}$, here $i=0,1, \cdots, g-2$. Then we get two graphs $\Gamma_{g}^{w}, \Gamma_{g}^{w^{\prime}} \in S^{3}$. $\Gamma_{g}^{w}$ contains $L_{1}$ and $\Gamma_{g}^{w^{\prime}}$ contains $L_{2}$. Each graph has $2 g-2$ 
vertices and $3 g-3$ edges. They are in the dual positions as in Figure $2(g=3)$, here graphs have been projected into the three dimensional Euclidean space $E^{3}$ from $S^{3}-\{(-1,0)\}$. The middle point of $a_{1} a_{3}$ is at the infinity.

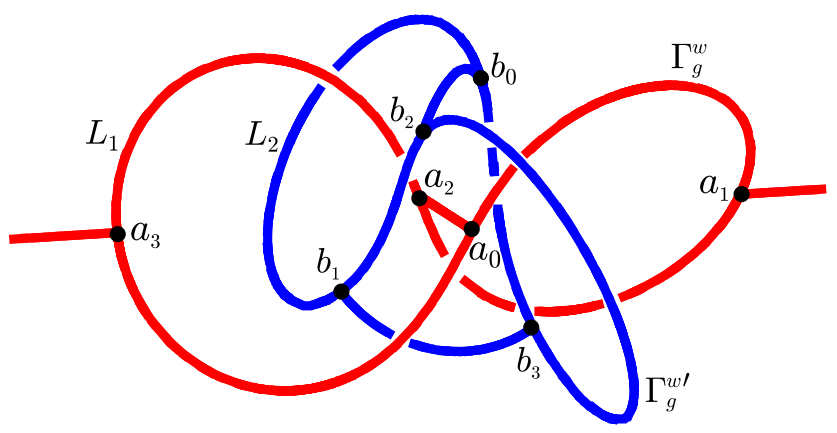

FiguRE 2. $\Gamma_{g}^{w}$ and $\Gamma_{g}^{w^{\prime}}$ in $S^{3}$

Let $T^{\prime}$ be the following torus in $S^{3}$ :

$$
T^{\prime}=\left\{\left(z_{1}, z_{2}\right) \in S^{3}|| z_{1}\left|=\frac{\sqrt{3}}{2},\right| z_{2} \mid=\frac{1}{2}\right\} .
$$

It splits $S^{3}$ into two solid tori $V_{1}{ }^{\prime}$ and $V_{2}{ }^{\prime}$ :

$$
\begin{aligned}
& V_{1}^{\prime}=\left\{\left(z_{1}, z_{2}\right) \in S^{3}|| z_{1} \mid \geq \frac{\sqrt{3}}{2}\right\}, \\
& V_{2}^{\prime}=\left\{\left(z_{1}, z_{2}\right) \in S^{3}|| z_{2} \mid \geq \frac{1}{2}\right\} .
\end{aligned}
$$

Let $D_{k}$ be the following meridian disk in $V_{1}{ }^{\prime}$ :

$$
D_{k}=\left\{\left(\sqrt{1-r^{2}} e^{\frac{\pi}{2 g-2} i+\frac{k \pi}{g-1} i}, r e^{\theta i}\right) \in S^{3} \mid 0 \leq r \leq \frac{1}{2}, \theta \in \mathbb{R}\right\} .
$$

It lies between $\left(e^{\frac{k \pi}{g-1} i}, 0\right)$ and $\left(e^{\frac{(k+1) \pi}{g-1} i}, 0\right)$, here $k=0,1, \ldots, 2 g-3$. Then these disks cut $V_{1}{ }^{\prime}$ into $2 g-2$ cylinders. And each cylinder intersects exactly one of $\Gamma_{g}^{w}$ and $\Gamma_{g}^{w^{\prime}}$. Let $A$ be the annulus in $V_{2}{ }^{\prime}$ separating $L_{1}$ and $L_{2}$ :

$$
A=\left\{\left(r e^{2 \theta i}, \sqrt{1-r^{2}} e^{\frac{\pi}{4} i+\theta i}\right) \in S^{3} \mid-\frac{\sqrt{3}}{2} \leq r \leq \frac{\sqrt{3}}{2}, \theta \in \mathbb{R}\right\} .
$$

Then $A$ cuts $V_{2}{ }^{\prime}$ into 2 solid tori. Let $V_{g}^{w}$ be the union of the solid torus and cylinders intersecting $\Gamma_{g}^{w}$, and $V_{g}^{w^{\prime}}$ be the union of the solid torus and cylinders intersecting $\Gamma_{g}^{w^{\prime}}$. Then $V_{g}^{w}$ and $V_{g}^{w^{\prime}}$ are two handlebodies. $\partial V_{g}^{w}=\partial V_{g}^{w^{\prime}} \cong \Sigma_{g}$, and $\partial V_{g}^{w}$ is a Heegaard surface of $S^{3}$.

Following isometry on $S^{3}$ preserves the graph $\Gamma_{g}^{w} \cup \Gamma_{g}^{w \prime}$, the torus $T^{\prime}$, the annulus $A$, and the union of the disks. It also preserves $\partial V_{g}^{w}$.

$$
\varphi_{g}:\left(z_{1}, z_{2}\right) \mapsto\left(e^{\frac{\pi}{g-1} i} z_{1}, i e^{\frac{\pi}{2 g-2} i} z_{2}\right)
$$

For odd $g>1, \varphi_{g}$ has order $4 g-4$. Notice that $\varphi_{g}$ preserves the orientation of $S^{3}$ and changes the two sides of $\partial V_{g}^{w}$. Combining with Lemma 2.1 for odd $g>1,\left\langle\varphi_{g}\right\rangle$ gives a type $(-,+)$ extendable cyclic group action of order $4 g-4$ on $\Sigma_{g} \cong \partial V_{g}^{w}$. This also realizes the maximum order of orientation-reversing periodic maps on $\Sigma_{g}$ when $g$ is odd. 
Example 2.4 (Fork). Given even $g>1$, we will construct an abelian group action on a Heegaard splitting of $S^{3}$.

Let $S^{3}$ be the unit sphere in $\mathbb{C}^{2}$ :

$$
S^{3}=\left\{\left.\left(z_{1}, z_{2}\right) \in \mathbb{C}^{2}|| z_{1}\right|^{2}+\left|z_{2}\right|^{2}=1\right\} .
$$

Let

$$
\begin{aligned}
& a_{m}=\left(e^{\frac{(2 m+1) \pi}{2} i}, 0\right), m=0,1, \\
& b_{n}=\left(0, e^{\frac{2 n \pi}{g+2} i}\right), n=0,1, \cdots, g+1 .
\end{aligned}
$$

Connect $a_{0}$ to each $b_{2 k}$ with the shortest geodesics in $S^{3}$, and connect $a_{1}$ to each $b_{2 k+1}$ with the shortest geodesics in $S^{3}$, here $k=0,1, \cdots, g / 2$. Then we get two graphs $\Gamma_{g}^{f}, \Gamma_{g}^{f^{\prime}} \in S^{3} . \Gamma_{g}^{f}$ contains $a_{0}, b_{2 k}$, and $\Gamma_{g}^{f^{\prime}}$ contains $a_{1}, b_{2 k+1}$. Let $S$ be the following two dimensional sphere in $S^{3}$ :

$$
S=\left\{\left(z_{1}, z_{2}\right) \in \mathbb{C}^{2} \mid z_{1} \in \mathbb{R}\right\} \cap S^{3} .
$$

It cuts $S^{3}$ into 2 three dimensional balls $B_{0}$ and $B_{1}$. $B_{0}$ contains $a_{0}$ and $B_{1}$ contains $a_{1} . \Gamma_{g}^{f}, \Gamma_{g}^{f^{\prime}}$ and $S$ are shown in Figure $3(g=4)$, here they have been projected into the three dimensional Euclidean space $E^{3}$ from $S^{3}-\{(-1,0)\}$.

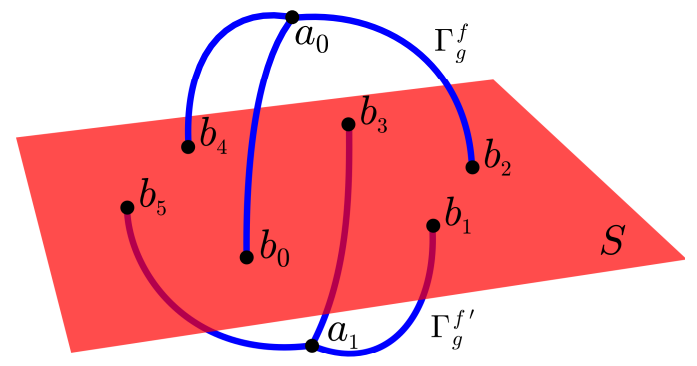

Figure $3 . \Gamma_{g}^{f}, \Gamma_{g}^{f^{\prime}}$ and $S$ in $S^{3}$

Following two isometries on $S^{3}$ preserve the sphere $S$ and the graph $\Gamma_{g}^{f} \cup \Gamma_{g}^{f^{\prime}}$.

$$
\begin{aligned}
\tau_{g+1}^{2}:\left(z_{1}, z_{2}\right) & \mapsto\left(-z_{1}, e^{\frac{2 \pi}{g+2} i} z_{2}\right) \\
\rho \sigma:\left(z_{1}, z_{2}\right) & \mapsto\left(-\bar{z}_{1}, z_{2}\right)
\end{aligned}
$$

Hence they also preserve some closed regular neighbourhood $N\left(\Gamma_{g}^{f}\right) \cup N\left(\Gamma_{g}^{f^{\prime}}\right)$ of $\Gamma_{g}^{f} \cup \Gamma_{g}^{f^{\prime}}$, and preserve the common boundary of the two handlebodies $V_{g}^{f}$ and $V_{g}^{f^{\prime}}$ :

$$
\begin{aligned}
V_{g}^{f} & =N\left(\Gamma_{g}^{f}\right) \cup \overline{B_{1}-N\left(\Gamma_{g}^{f^{\prime}}\right)}, \\
V_{g}^{f^{\prime}} & =N\left(\Gamma_{g}^{f^{\prime}}\right) \cup \overline{B_{0}-N\left(\Gamma_{g}^{f}\right) .}
\end{aligned}
$$

The surface $\partial V_{g}^{f}=\partial V_{g}^{f^{\prime}}$ is a Heegaard surface of $S^{3}$, and $\partial V_{g}^{f} \cong \Sigma_{g}$.

Since $\tau_{g+1}^{2} \rho \sigma=\rho \sigma \tau_{g+1}^{2},\left\langle\tau_{g+1}^{2}, \rho \sigma\right\rangle$ is an abelian group of order $2 g+4$. Since $\tau_{g+1}^{2}$ preserves the orientation of $S^{3}, \rho \sigma$ reverses the orientation of $S^{3}$, and only $\tau_{g+1}^{2}$ changes the two sides of $\partial V_{g}^{f}$, combining with Lemma 2.1, one can check that $\tau_{g+1}^{2} \rho \sigma$ and $\tau_{g+1}^{2}$ satisfy the condition of type (Mix) extendable group action. Hence for even $g>1,\left\langle\tau_{g+1}^{2}, \rho \sigma\right\rangle$ gives a type (Mix) extendable abelian group action of order $2 g+4$ on $\Sigma_{g} \cong \partial V_{g}^{f}$. 
Example 2.5 (Square). We will construct a $\oplus_{i=1}^{5} \mathbb{Z}_{2}$-action on $V_{5}$.

Let $\Gamma^{s}$ be the boundary of the square $[0,1]^{2}$ in $x y$-plane in the three dimensional Euclidean space $E^{3}$. Let $T_{r}$ and $T_{r}{ }^{\prime} \subset T_{r}$ be following translation groups:

$$
\begin{aligned}
T_{r} & =\{(a, b, c) \mid a, b, c \in \mathbb{Z}\}, \\
T_{r}{ }^{\prime} & =\langle(2,0,0),(0,2,0),(0,0,1)\rangle .
\end{aligned}
$$

An element $t=(a, b, c) \in T_{r}$ acts on $E^{3}$ as following:

$$
t:(x, y, z) \mapsto(x+a, y+b, z+c) .
$$

Following three isometries $r_{x}, r_{y}$ and $R_{z}$ on $E^{3}$ preserve the graph $\bigcup_{t \in T_{r}} t\left(\Gamma^{s}\right)$.

$$
\begin{aligned}
r_{x}:(x, y, z) & \mapsto(x,-y,-z) \\
r_{y}:(x, y, z) & \mapsto(-x, y,-z) \\
R_{z}:(x, y, z) & \mapsto(x, y,-z)
\end{aligned}
$$

In the three dimensional torus $E^{3} / T_{r}{ }^{\prime} \cong T^{3}$ we have a graph $\Gamma_{5}^{s}=\left(\bigcup_{t \in T_{r}} t\left(\Gamma^{s}\right)\right) / T_{r}{ }^{\prime}$. It has 4 vertices and 8 edges. Since $T_{r}{ }^{\prime}$ is a normal subgroup of $\left\langle T_{r}, r_{x}, r_{y}, R_{z}\right\rangle$, the quotient group $\left\langle T_{r}, r_{x}, r_{y}, R_{z}\right\rangle / T_{r}{ }^{\prime} \cong \oplus_{i=1}^{5} \mathbb{Z}_{2}$ acts on $E^{3} / T_{r}{ }^{\prime}$, and it preserves $\Gamma_{5}^{s}$. Then it also preserves some closed regular neighbourhood $N\left(\Gamma_{5}^{s}\right) \cong V_{5}$ of $\Gamma_{5}^{s}$.

We see this $\oplus_{i=1}^{5} \mathbb{Z}_{2}$-action on $V_{5}$ is "extendable over $T^{3}$ ". But even on $\partial V_{5} \cong \Sigma_{5}$, it is not extendable over $S^{3}$, by Theorem 1.2 ,

\section{Preliminaries For the Proofs}

In this section we will give some known results about actions of discrete groups on surfaces and three manifolds, which will be used later. Most results will be presented in the language of orbifold.

Remark 3.1. For orbifold theories, one can see [1, 7, 12, 15]. In the following, contents in 3.1 can be found in [1. Lemma 3.6 can be found in 3]; Lemma 3.7 and 3.8 rely on 1, 2, 5, 10, 12, 5] for the orbifold having isolated singular points, and 1, 2, 10, 12 for the orbifold whose singular set has dimension at least 1; Lemma 3.9, 3.14 and 3.15 can be found in [7, 15]; Lemma 3.16 can be found in [9].

3.1. Basic concepts in orbifold theory. Let $M$ be a n-dimensional manifold with a faithful smooth action of a discrete group $G$, then $M / G$ is an n-orbifold. For any $x \in M / G$, let $x^{\prime}$ be one of its pre-images in $M$, and $S t\left(x^{\prime}\right)$ be the stable subgroup of $x^{\prime}$. Then the isomorphic type of $S t\left(x^{\prime}\right)$ does not depend on the choice of $x^{\prime}$. Denote it by $G_{x}$. It is the local group of $M / G$ at $x$. The order of $G_{x}$ is the index of $x$. If $\left|G_{x}\right|>1, x$ is a singular point of $M / G$. Otherwise $x$ is a regular point of $M / G$. If we forget all local groups and indices, then we get the underlying space $|M / G|$, which is a topological space with the quotient topology.

The orbifold $M / G$ is orientable if $M$ is orientable and $G$ preserves the orientation of $M . M / G$ is connected if $|M / G|$ is connected. And $M / G$ is compact if $|M / G|$ is compact. $M / G$ has non-empty boundary $\partial M / G$ if $M$ has non-empty boundary $\partial M$, then $\partial M / G$ is the orbifold $(\partial M) / G . M / G$ is closed if it is connected, compact and has empty boundary.

Two orbifolds $M_{1} / G_{1}$ and $M_{2} / G_{2}$ are homeomorphic via $p: M_{1} / G_{1} \rightarrow M_{2} / G_{2}$ if the induced map $\left|M_{1} / G_{1}\right| \rightarrow\left|M_{2} / G_{2}\right|$ is a homeomorphism, $p$ preserves local groups, and at any point $p$ can be locally lifted to an equivariant homeomorphism between open sets of $M_{1}$ and $M_{2}$. 
If $M / G \cong M^{\prime} / G^{\prime}$ and $M^{\prime}$ is simply connected, then the fundamental group $\pi_{1}(M / G) \cong G^{\prime}$. Covering spaces of $M / G$ can be defined, and there is a one to one correspondence between covering spaces and conjugacy classes of subgroups of $\pi_{1}(M / G)$. Regular covering spaces will correspond to normal subgroups. A similar Van-Kampen theorem is also valid.

Definition 3.2. Let $P_{T}$ (respectively $P_{O}, P_{I}$ ) be a regular tetrahedron (respectively octahedron, icosahedron) centered at $(0,0,0)$ in $E^{3}$. For a polyhedron $P$ in $E^{3}$, define $I^{+}(P)$ to be the orientation-preserving isometric group of $P$.

For $n \in \mathbb{Z}_{+}$, define $r_{n}$ to be the following isometry on $E^{3}$ :

$$
r_{n}:(x, y, z) \mapsto\left(x \cos \frac{2 \pi}{n}+y \sin \frac{2 \pi}{n},-x \sin \frac{2 \pi}{n}+y \cos \frac{2 \pi}{n}, z\right) .
$$

Lemma 3.3. Let $x$ be a point in an orientable 2-orbifold, then $x$ has a neighbourhood which is homeomorphic to some $E^{2} /\left\langle r_{n}\right\rangle$, and $G_{x} \cong\left\langle r_{n}\right\rangle, n \in \mathbb{Z}_{+}$. Here $E^{2}$ is the $x y$-plane in $E^{3}$.

Lemma 3.4. Let $x$ be a point in an orientable 3-orbifold, then $x$ has a neighbourhood which is homeomorphic to some $E^{3} / H$, and $G_{x} \cong H$. H is one of the groups $\left\langle r_{n}\right\rangle,\left\langle r_{n}, r_{x}\right\rangle, I^{+}\left(P_{T}\right), I^{+}\left(P_{O}\right), I^{+}\left(P_{I}\right), n \in \mathbb{Z}_{+}$. Here $r_{x}$ is defined in Example 2.5

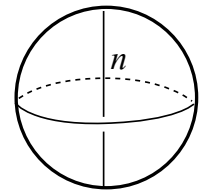

cyclic

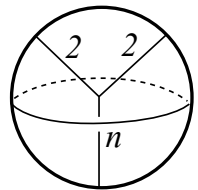

dihedral

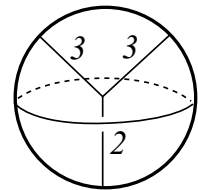

tetrahedral

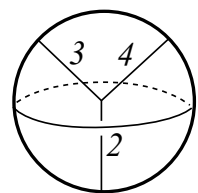

octahedral

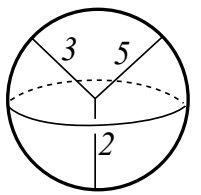

icosahedral

Figure 4. Local models of 3-orbifolds

\subsection{Results about discal, spherical and handlebody orbifolds.}

Definition 3.5. Let $B^{n}$ and $S^{n}$ be the n-ball and n-sphere respectively. An orbifold which is homeomorphic to some $B^{n} / G$ (respectively $S^{n} / G, V_{g} / G$ ) is called a discal n-orbifold (respectively spherical n-orbifold, handlebody orbifold).

Lemma 3.6. An orientable discal 2-orbifold is homeomorphic to some $B_{u}^{2} /\left\langle r_{n}\right\rangle$. Here $B_{u}^{2}$ is the unit 2-ball centered at $(0,0,0)$ in the xy-plane.

Lemma 3.7. An orientable discal 3-orbifold is homeomorphic to some $B_{u}^{3} / H$, and an orientable spherical 2-orbifold is homeomorphic to some $S_{u}^{2} / H$. Here $B_{u}^{3}$ and $S_{u}^{2}$ are the unit 3-ball and 2-sphere centered at $(0,0,0)$ in $E^{3}$ respectively. $H$ is one of $\left\langle r_{n}\right\rangle,\left\langle r_{n}, r_{x}\right\rangle, I^{+}\left(P_{T}\right), I^{+}\left(P_{O}\right), I^{+}\left(P_{I}\right), n \in \mathbb{Z}_{+}$.

Lemma 3.8. Let $S^{3} / G$ be a spherical orbifold, here $G$ is a non-trivial cyclic group. If $S^{3} / G$ is orientable, then the set of singular points of index $|G|$ is $\emptyset$ or a $S^{1}$.

If $S^{3} / G$ is not orientable, then the set of singular points of index $|G|$ is a $S^{0}$ or a $S^{2}$. Here $S^{0} \cong\{0,1\}$ with discrete topology.

Lemma 3.9. An orientable handlebody orbifold is a union of finitely many orientable discal 3-orbifolds $\left\{B_{i}^{3} / G_{i}\right\}_{i=1}^{n}, n \in \mathbb{Z}_{+}$, such that:

(a). In each $\partial B_{i}^{3} / G_{i}$ finitely many disjoint orientable discal 2-orbifolds are given; 
(b). For $i \neq j$, if $B_{i}^{3} / G_{i} \cap B_{j}^{3} / G_{j} \neq \emptyset$, then $B_{i}^{3} / G_{i} \cap B_{j}^{3} / G_{j}$ is a union of finitely many orientable discal 2-orbifolds in (a);

(c). Each discal 2-orbifold in (a) is in exactly two elements in $\left\{B_{i}^{3} / G_{i}\right\}_{i=1}^{n}$.

(d). There is a choice of orientations of $\left\{B_{i}^{3} / G_{i}\right\}_{i=1}^{n}$, such that on any common discal 2-orbifold, the induced orientations are opposite.

Conversely, if a connected orientable 3-orbifold is a union of finitely many orientable discal 3-orbifolds $\left\{B_{i}^{3} / G_{i}\right\}_{i=1}^{n}$ satisfying $(a-d)$, then it is an orientable handlebody orbifold. $\bigcup_{i=1}^{n} B_{i}^{3} / G_{i}$ is called a discal orbifold decomposition of it.

Definition 3.10. A finite graph of finite groups $\mathcal{G}$ is a labelled finite graph. Each vertex and edge is labelled by a finite group. If $G_{v}$ is a vertex adjacent to an edge $G_{e}$, then there is an injective homomorphism $\phi_{e v}: G_{e} \rightarrow G_{v}$. If we forget all labels, then we get its underlying graph $|\mathcal{G}|$.

Definition 3.11. Let $\mathcal{H}$ be an orientable handlebody orbifold. $\mathcal{H}=\bigcup_{i=1}^{n} B_{i}^{3} / G_{i}$ is a discal orbifold decomposition as in Lemma 3.9. Then $\mathcal{H}$ has an induced finite graph of finite groups (induced by the discal orbifold decomposition) defined as following: it contains $\left\{G_{i}\right\}_{i=1}^{n}$ as vertices; there is an edge $G$ between $G_{i}$ and $G_{j}$ if $B_{i}^{3} / G_{i}$ and $B_{j}^{3} / G_{j}$ share a common discal 2-orbifold $B^{2} / G$.

Definition 3.12. Let $\mathcal{G}$ be a finite graph of finite groups. $V$ and $E$ are its vertex set and edge set respectively. Define its Euler characteristic:

$$
\chi(\mathcal{G})=\sum_{G_{v} \in V} \frac{1}{\left|G_{v}\right|}-\sum_{G_{e} \in E} \frac{1}{\left|G_{e}\right|} .
$$

Let $\mathcal{H}$ be an orientable handlebody orbifold, and $\mathcal{H} \cong V_{g} / G$. Define its Euler characteristic:

$$
\chi(\mathcal{H})=\frac{1-g}{|G|} .
$$

Definition 3.13. A homomorphism $\phi: H \rightarrow G$ between groups is a finitely injective surjection if it is surjective and on any finite subgroup of $H$ it is injective.

Lemma 3.14. Let $\mathcal{H}$ be an orientable handlebody orbifold. Let $\mathcal{G}$ be an induced finite graph of finite groups of $\mathcal{H}$. Then $\chi(\mathcal{H})=\chi(\mathcal{G})$.

Lemma 3.15. Let $\mathcal{H}$ be an orientable handlebody orbifold, and $G$ be a finite group. Then $G$ can orientation-preservingly act on $V_{g}$ such that $V_{g} / G \cong \mathcal{H}$ if and only if there is a finitely injective surjection $\phi: \pi_{1}(\mathcal{H}) \rightarrow G$ and $1-g=\chi(\mathcal{H})|G|$.

Lemma 3.16. Let $G$ be an abelian group acting on $\Sigma_{g}$. Suppose $\Sigma_{g} / G$ is orientable, having singular points $p_{1}, \cdots, p_{\alpha}$ and $q_{1}, \cdots, q_{\beta}$. Index of $p_{j}(1 \leq j \leq \alpha)$ is $n_{j}$ and $n_{j}>2$; index of $q_{k}(1 \leq k \leq \beta)$ is 2 . Then for some $\gamma \in \mathbb{Z}_{+} \cup\{0\}$ we have

$$
\begin{aligned}
\pi_{1}(\Sigma / G)= & \left\langle a_{1}, b_{1}, \cdots, a_{\gamma}, b_{\gamma}, x_{1}, \cdots, x_{\alpha}, y_{1}, \cdots, y_{\beta}\right| \\
& \left.\prod_{i=1}^{\gamma}\left[a_{i}, b_{i}\right] \prod_{j=1}^{\alpha} x_{j} \prod_{k=1}^{\beta} y_{k}=1, x_{j}^{n_{j}}=y_{k}^{2}=1,1 \leq j \leq \alpha, 1 \leq k \leq \beta\right\rangle .
\end{aligned}
$$

The action corresponds to a finitely injective surjection $\phi: \pi_{1}\left(\Sigma_{g}\right) \rightarrow G$. Then the following (a), (b) and (c) are equivalent:

(a). The $G$-action on $\Sigma_{g}$ extends to a handlebody $V_{g}$, with $\partial V_{g}=\Sigma_{g}$.

(b). The $G$-action on $\Sigma_{g}$ extends to a compact 3-manifold $M$, with $\partial M=\Sigma_{g}$.

(c). The generators $x_{1}, \cdots, x_{\alpha}$ corresponding to $p_{1}, \cdots, p_{\alpha}$ can be partitioned into pairs $x_{s}, x_{t}$ such that $\phi\left(x_{s}\right)=\phi\left(x_{t}^{-1}\right)$. 


\section{MAXIMUM ORDERS OF CYCLIC AND ABELIAN ACTIONS ON HANDLEBODIES}

In this section we will determine the maximum orders of cyclic and abelian group actions on orientable handlebodies. Firstly we need some lemmas.

\subsection{Some useful lemmas.}

Lemma 4.1. Let $V_{g} / G$ be an orientable handlebody orbifold. Here $G$ is cyclic and $|G|>g-1$. Then $V_{g} / G$ has an induced finite graph of finite groups as one of the following, here $l, m, n>1$.

(A). $\quad \mathbb{Z}_{l}-\mathbb{Z}_{m}-\mathbb{Z}_{n} \quad(1 / l+1 / m+1 / n>1)$

(B). $\quad \mathbb{Z}_{m}-\mathbb{Z}_{n} \quad(0<1 / m+1 / n<1)$

(C). $\quad \mathbb{Z}_{n} \bigcirc$

(D). $\quad \mathbb{Z}_{n} \longrightarrow \mathbb{Z}_{m} \supset \mathbb{Z}_{m}$

Proof. Let $V_{g} / G=\bigcup_{i=1}^{n} B_{i}^{3} / G_{i}$ as in Lemma 3.9. and $\mathcal{G}$ be the induced finite graph of finite groups. Since $|G|>g-1$, by Definition 3.12 and Lemma 3.14 we have:

$$
\chi(\mathcal{G})=\chi\left(V_{g} / G\right)=\frac{1-g}{|G|} \in(-1,0) .
$$

Since $G$ is cyclic, all the vertices and edges of $\mathcal{G}$ are cyclic groups. Hence if an edge $G_{e}$ is non-trivial, then any vertex of it must be isomorphic to it, and if a vertex $G_{v}$ is non-trivial, then there are at most two non-trivial edges adjacent to it. Let $V$ and $E$ be the vertex set and edge set of $\mathcal{G}$ respectively, and $V^{\prime}$ and $E^{\prime}$ be the non-trivial vertex set and edge set of $\mathcal{G}$ respectively. By Definition 3.12 we have:

$$
\begin{aligned}
\chi(\mathcal{G}) & =\chi(|\mathcal{G}|)+\sum_{G_{v} \in V}\left(\frac{1}{\left|G_{v}\right|}-1\right)-\sum_{G_{e} \in E}\left(\frac{1}{\left|G_{e}\right|}-1\right) \\
& =\chi(|\mathcal{G}|)+\sum_{G_{v} \in V^{\prime}}\left(\frac{1}{\left|G_{v}\right|}-1\right)-\sum_{G_{e} \in E^{\prime}}\left(\frac{1}{\left|G_{e}\right|}-1\right) \\
& <\chi(|\mathcal{G}|) .
\end{aligned}
$$

Hence $\chi(|\mathcal{G}|) \in\{0,1\}$.

If $\chi(|\mathcal{G}|)=1$, then $|\mathcal{G}|$ is a tree. If $\mathcal{G}$ contains a trivial vertex $G_{i}$, and $G_{e}$ is an edge adjacent to $G_{v}, G_{j}$ is the other vertex of $G_{e}$, then $G_{e}$ is trivial; if $\mathcal{G}$ contains a non-trivial edge $G_{e}$, and $G_{i}, G_{j}$ are its two vertices, then $G_{e} \cong G_{i} \cong G_{j}$. In each case $B_{i}^{3} / G_{i} \cup B_{j}^{3} / G_{j} \cong B_{j}^{3} / G_{j}$. Replacing $B_{i}^{3} / G_{i}$ and $B_{j}^{3} / G_{j}$ by their union, we get a new discal decomposition of $V_{g} / G$. The new induced finite graph of finite groups will have less trivial vertices or less non-trivial edges. Hence we can assume $V^{\prime}=V$ and $E^{\prime}=\emptyset$. Then

$$
\chi(\mathcal{G})=1+\sum_{G_{v} \in V^{\prime}}\left(\frac{1}{\left|G_{v}\right|}-1\right) .
$$

Since for $G_{v} \in V^{\prime}$ we have $\left|G_{v}\right|>1,\left|V^{\prime}\right|=|V|$ must be 2 or 3 . Hence $\mathcal{G}$ will be some finite graph of finite groups in the class (A) or (B).

If $\chi(|\mathcal{G}|)=0$, then we can delete an edge $G_{e}$ of $\mathcal{G}$ to get a maximal tree $\mathcal{G}^{\prime}$ of it. Similar to the above discussion, we can assume $V^{\prime}=V$ and edges in $\mathcal{G}^{\prime}$ are trivial. 
The edge $G_{e}$ may be trivial or non-trivial. Then

$$
\chi(\mathcal{G})=\sum_{G_{v} \in V^{\prime}}\left(\frac{1}{\left|G_{v}\right|}-1\right)-\left(\frac{1}{\left|G_{e}\right|}-1\right) .
$$

If $G_{e}$ is trivial, then $\left|V^{\prime}\right|=|V|=1$. Hence $\mathcal{G}$ will be some finite graph of finite groups in the class (C). If $G_{e}$ is non-trivial, then $\left|V^{\prime}\right|=|V|=2$. If $G_{e}$ is a loop, then $\mathcal{G}$ will be some finite graph of finite groups in the class (D). Otherwise, as above we can get a new $\mathcal{G}$ with less non-trivial edges. Then $\mathcal{G}$ will be some finite graph of finite groups in the class $(\mathrm{C})$.

Let $\mathcal{C}$ be one of the classes (A-D) in Lemma 4.1. In the following, we say that an orientable handlebody orbifold $\mathcal{H}$ is in the class $\mathcal{C}$ if $\mathcal{H}$ has an induced finite graph of finite groups belonging to $\mathcal{C}$. Following Lemma 4.2 and 4.3 can be derived easily from the Van-Kampen theorem and Definition 3.12 .

Lemma 4.2. The fundamental groups of the handlebody orbifolds in classes ( $A-D)$ are given in Table 4 .

TABLE 4. Fundamental groups of classes (A-D)

\begin{tabular}{|c|l|}
\hline$(A)$ & $\mathbb{Z}_{l} * \mathbb{Z}_{m} * \mathbb{Z}_{n}$ \\
\hline$(B)$ & $\mathbb{Z}_{m} * \mathbb{Z}_{n}$ \\
\hline$(C)$ & $\mathbb{Z}_{n} * \mathbb{Z}$ \\
\hline$(D)$ & $\mathbb{Z}_{n} *\left(\mathbb{Z}_{m} \oplus \mathbb{Z}\right)$ \\
\hline
\end{tabular}

Lemma 4.3. The Euler characteristic of the finite graphs of finite groups in classes $(A-D)$ are given in Table 5 .

TABLE 5. Euler characteristic of classes (A-D)

\begin{tabular}{|l|l|}
\hline$(A)$ & $1 / l+1 / m+1 / n-2$ \\
\hline$(B)$ & $1 / m+1 / n-1$ \\
\hline$(C)$ & $1 / n-1$ \\
\hline$(D)$ & $1 / n-1$ \\
\hline
\end{tabular}

Lemma 4.4. Let $\langle h\rangle$ be a finite cyclic group acting faithfully on a manifold $M . \bar{h}$ is the image of $h$ in $\langle h\rangle /\left\langle h^{2}\right\rangle$. Then $\bar{h}$ acts on $M /\left\langle h^{2}\right\rangle$. Suppose $x$ is a fixed point of $\bar{h}$ and $x$ has index $\left|G_{x}\right|$ in $M /\left\langle h^{2}\right\rangle$, then $\left|\left\langle h^{2}\right\rangle\right| /\left|G_{x}\right|$ is odd.

Proof. Let $x^{\prime}$ be a pre-image of $x$ in $M$. Since $x$ is a fixed point of $\bar{h}$, the orbit $\left\{h^{i}\left(x^{\prime}\right) \mid i \in \mathbb{Z}\right\}$ and $\left\{h^{2 i}\left(x^{\prime}\right) \mid i \in \mathbb{Z}\right\}$ are the same. Hence the pre-image of $x$ in $M$ contains odd points. Namely $\left|\left\langle h^{2}\right\rangle\right| /\left|G_{x}\right|$ is odd.

Lemma 4.5. Let $h$ be a periodic map of order 2 on a compact manifold $M$. If the Euler characteristic of $M$ is odd, then $h$ has a fixed point.

Proof. Since $h$ has order 2 , the induced linear map $h_{* i}: H_{i}(M, \mathbb{R}) \rightarrow H_{i}(M, \mathbb{R})$ can only have eigenvalues \pm 1 . Let $L(h)$ be the Lefschetz number of $h$, and $\chi(M)$ be the Euler characteristic of $M$. Then $L(h) \equiv \chi(M)(\bmod 2)$. Since $\chi(M)$ is odd, $L(h) \neq 0$. Then by the Lefschetz fixed point theorem, $h$ has a fixed point. 
Lemma 4.6. Let $l, m, n \in \mathbb{Z}_{+}$, and for $1 \leq i \leq l, m_{i} \in \mathbb{Z}_{+}$. Let $\left[m_{1}, m_{2}, \cdots, m_{l}\right]$ be the lowest common multiple of $m_{i}, 1 \leq i \leq l$.

(1). If $\phi: \mathbb{Z}_{m_{1}} * \mathbb{Z}_{m_{2}} * \cdots * \mathbb{Z}_{m_{l}} \rightarrow \mathbb{Z}_{n}$ is a finitely injective surjection, then $n=\left[m_{1}, m_{2}, \cdots, m_{l}\right]$.

(2). If $\phi: \mathbb{Z}_{m_{1}} * \mathbb{Z}_{m_{2}} * \cdots * \mathbb{Z}_{m_{l}} *\left(\mathbb{Z}_{m} \oplus \mathbb{Z}\right) \rightarrow \mathbb{Z}_{n}$ is a finitely injective surjection, then $n=t\left[m_{1}, m_{2}, \cdots, m_{l}, m\right]$ for some $t \in \mathbb{Z}_{+}$.

Proof. (1). The homomorphism $\phi$ induces a surjective homomorphism

$$
\phi^{\prime}: \mathbb{Z}_{m_{1}} \oplus \mathbb{Z}_{m_{2}} \oplus \ldots \oplus \mathbb{Z}_{m_{l}} \rightarrow \mathbb{Z}_{n}
$$

which is injective on $\mathbb{Z}_{m_{i}}, 1 \leq i \leq l$. For any prime number $p$, let $\mathbb{Z}_{p^{\alpha_{i}}}$ be the $p$-component of $\mathbb{Z}_{m_{i}}$, and $\mathbb{Z}_{p^{\beta}}$ be the $p$-component of $\mathbb{Z}_{n}$. Since $\phi^{\prime}$ is surjective, we have $\max \left\{\alpha_{1}, \alpha_{2}, \cdots, \alpha_{l}\right\} \geq \beta$. Otherwise generators of $\mathbb{Z}_{p^{\beta}}$ will not lie in the image of $\phi^{\prime}$. Since $\phi^{\prime}$ is injective on $\mathbb{Z}_{m_{i}}$, we have $\beta \geq \max \left\{\alpha_{1}, \alpha_{2}, \cdots, \alpha_{l}\right\}$. Hence $\beta=\max \left\{\alpha_{1}, \alpha_{2}, \cdots, \alpha_{l}\right\}$, namely $n=\left[m_{1}, m_{2}, \cdots, m_{l}\right]$.

(2). The homomorphism $\phi$ induces a surjective homomorphism

$$
\phi^{\prime}: \mathbb{Z}_{m_{1}} \oplus \mathbb{Z}_{m_{2}} \oplus \ldots \oplus \mathbb{Z}_{m_{l}} \oplus\left(\mathbb{Z}_{m} \oplus \mathbb{Z}\right) \rightarrow \mathbb{Z}_{n},
$$

which is injective on $\mathbb{Z}_{m}$ and $\mathbb{Z}_{m_{i}}, 1 \leq i \leq l$. Let $\mathbb{Z}_{p^{\alpha}}$ be the $p$-component of $\mathbb{Z}_{m}$, then $\beta \geq \max \left\{\alpha_{1}, \cdots, \alpha_{l}, \alpha\right\}$. Hence $n=t\left[m_{1}, \cdots, m_{l}, m\right]$ for some $t \in \mathbb{Z}_{+}$.

\subsection{Maximum orders of cyclic and abelian actions on handlebodies.}

Proposition 4.7. Table 3 gives an upper bound of $\mathrm{CH}_{g}^{-}$.

Proof. Let $\langle h\rangle$ be a cyclic group acting on $V_{g} . h$ reverses the orientation of $V_{g}$. Let $\bar{h}$ be the image of $h$ in $\langle h\rangle /\left\langle h^{2}\right\rangle$. Then $\bar{h}$ acts on $V_{g} /\left\langle h^{2}\right\rangle$ and has order 2.

By (2) and $\left(2^{\prime}\right)$ in Example 2.2 we have $\mathrm{CH}_{g}^{-}>2 g-2$. If $|\langle h\rangle|=C H_{g}^{-}$, then $\left|\left\langle h^{2}\right\rangle\right|>g-1$. By Lemma 4.1, $V_{g} /\left\langle h^{2}\right\rangle$ is in classes (A-D). Let $\Theta$ be the set of singular points in $V_{g} /\left\langle h^{2}\right\rangle$, and $N(\Theta)$ be a $\bar{h}$-invariant regular neighborhood of $\Theta$. Then $\bar{h}$ acts on $\Theta$ and $\overline{\left(V_{g} /\left\langle h^{2}\right\rangle\right) \backslash N(\Theta)}$ respectively.

Case 1: $V_{g} /\left\langle h^{2}\right\rangle$ is in the class (A).

Then $\left|\left\langle h^{2}\right\rangle\right|$ is even. By Lemma 4.5, $\bar{h}$ will have a fixed point in $\Theta$. Then by Lemma 4.4. for a fixed point $x$ we have that $\left|\left\langle h^{2}\right\rangle\right| /\left|G_{x}\right|$ is odd. Hence the index of $x$ must be even. Then we have $\{l, m, n\}=\{2,3,3\}$ or $\{2,2,2 k\}, k \in \mathbb{Z}_{+}$.

If $\{l, m, n\}=\{2,3,3\}$, then by Lemma 3.15, there is a finitely injective surjection $\phi: \pi_{1}\left(V_{g} /\left\langle h^{2}\right\rangle\right) \rightarrow\left\langle h^{2}\right\rangle$. By Lemma 4.2 and 4.6, $\pi_{1}\left(V_{g} /\left\langle h^{2}\right\rangle\right) \cong \mathbb{Z}_{2} * \mathbb{Z}_{3} * \mathbb{Z}_{3}$ and $\left\langle h^{2}\right\rangle \cong \mathbb{Z}_{6}$. Hence $|\langle h\rangle|=12$. Then by Lemma 4.3, we have $g=6$.

Similarly, if $\{l, m, n\}=\{2,2,2 k\}$, then $\left\langle h^{2}\right\rangle \cong \mathbb{Z}_{2 k},|\langle h\rangle|=4 k$ and $g=2 k$.

Case 2: $V_{g} /\left\langle h^{2}\right\rangle$ is in the classes (B-D).

By Lemma 4.5, $\bar{h}$ will have a fixed point $x$ in $\overline{\left(V_{g} /\left\langle h^{2}\right\rangle\right) \backslash N(\Theta)}$. By Lemma 4.4 $\left|\left\langle h^{2}\right\rangle\right| /\left|G_{x}\right|=\left|\left\langle h^{2}\right\rangle\right|$ is odd. Then by Lemma 3.15, 4.2, 4.6 and 4.3, we have:

If $V_{g} /\left\langle h^{2}\right\rangle$ is in the class (B), then $\pi_{1}\left(V_{g} /\left\langle h^{2}\right\rangle\right) \cong \mathbb{Z}_{m} * \mathbb{Z}_{n}$, and $\left\langle h^{2}\right\rangle \cong \mathbb{Z}_{[m, n]}$. Then $|\langle h\rangle|=2[m, n]$, and $g=[m, n]-(m+n) /(m, n)+1$. Since $n$ and $m$ are odd, $g$ is even.

If $V_{g} /\left\langle h^{2}\right\rangle$ is in the class (C), then $\pi_{1}\left(V_{g} /\left\langle h^{2}\right\rangle\right) \cong \mathbb{Z}_{n} * \mathbb{Z}$, and $\left\langle h^{2}\right\rangle \cong \mathbb{Z}_{k n}$. Then $|\langle h\rangle|=2 k n$, and $g=k n-k+1$. Since $k$ and $n$ are odd, $g$ is odd.

If $V_{g} /\left\langle h^{2}\right\rangle$ is in the class (D), then $\pi_{1}\left(V_{g} /\left\langle h^{2}\right\rangle\right) \cong \mathbb{Z}_{n} *\left(\mathbb{Z}_{m} \oplus \mathbb{Z}\right)$, and we have $\left\langle h^{2}\right\rangle \cong \mathbb{Z}_{k[m, n]}$. Then $|\langle h\rangle|=2 k[m, n]$, and $g=k[m, n]-k[m, n] / n+1$. Since $k$, $m$ and $n$ are odd, $g$ is odd. Let $m=m^{\prime}(m, n)$, then $|\langle h\rangle|=2\left(k m^{\prime}\right) n$, and we have $g=\left(k m^{\prime}\right) n-k m^{\prime}+1$. 
Proposition 4.8. Suppose $k, m, n \in \mathbb{Z}_{+}$. If $g=[m, n]-(m+n) /(m, n)+1$, then there is a cyclic group $\langle h\rangle$ of order $2[m, n]$ acting on $V_{g}$. And $h$ reverses the orientation of $V_{g}$.

If $g=k n-k+1$, then there is a cyclic group $\langle h\rangle$ of order $2 k n$ acting on $V_{g}$. And $h$ reverses the orientation of $V_{g}$.

Proof. We can assume $m, n>1$. For $g=[m, n]-(m+n) /(m, n)+1$, let $\mathcal{F}$ be a 2-orbifold. It has underlying space $B^{2}$ and contains two singular points of indices $m$ and $n$ in the interior of $B^{2}$. Let $\mathcal{H}=\mathcal{F} \times[-1,1]$. It is in the class (B). $\pi_{1}(\mathcal{H})=\mathbb{Z}_{m} * \mathbb{Z}_{n}$, and there is a finitely injective surjection $\phi: \pi_{1}(\mathcal{H}) \rightarrow \mathbb{Z}_{[m, n]}$. Hence by Lemma 3.15, there is a $\mathbb{Z}_{[m, n]}$-action on $V_{g}$ such that $V_{g} / \mathbb{Z}_{[m, n]} \cong \mathcal{H}$.

The pre-image of $\mathcal{F} \times\{0\}$ in $V_{g}$ is a surface $F$ and $V_{g}=F \times[-1,1]$. Then the product $I d_{F} \times\left(-I d_{[-1,1]}\right)$ acts of $V_{g}$ and has order 2. It is commutative with the $\mathbb{Z}_{[m, n]}$-action. Hence we have a cyclic action of order $2[m, n]$ on $V_{g}$. Its generator reverses the orientation of $V_{g}$.

For $g=k n-k+1$, the construction is similar. In this case $\mathcal{F}$ has underlying space an annulus and contains one singular point of index $n$ in its interior. $\mathcal{H}=\mathcal{F} \times[-1,1]$ is in the class $(\mathrm{C})$.

Proposition 4.9. $A H_{g}^{-}=2 A H_{g}$.

Proof. Clearly $A H_{g}^{-} \leq 2 A H_{g}$. By (6) in Example 2.2 and Example 2.5 we have $A H_{g}^{-} \geq 2 A H_{g}$.

Proposition 4.10. $A_{g}^{-}=A H_{g}^{-}$.

Proof. Let $G$ be an abelian group acting on $\Sigma_{g} . \Sigma_{g} / G$ is not orientable. Let $G_{o}$ be the subgroup of $G$ containing all elements which preserve the orientation of $\Sigma_{g}$. Then we have a 2-sheet cover $\Sigma_{g} / G_{O} \rightarrow \Sigma_{g} / G$, and the covering transformation $\tau$ reverses the orientation of $\Sigma_{g} / G_{o}$.

Let $\left\{p_{1}, \cdots, p_{\alpha}, q_{1}, \cdots, q_{\beta}\right\}$ be the set of singular points of $\Sigma_{g} / G_{o} . p_{j}(1 \leq j \leq \alpha)$ has index bigger than 2 , and $q_{k}(1 \leq k \leq \beta)$ has index 2 . Then $p_{1}, \cdots, p_{\alpha}$ can be partitioned into pairs by $\tau$. Since $G$ is abelian and $\tau$ reverses the orientation of $\Sigma_{g} / G_{o}$, their corresponding generators $x_{1}, \cdots, x_{\alpha}$ in $\Sigma_{g} / G_{o}$ can be partitioned into pairs satisfying the condition of Lemma 3.16. Then $G_{o}$ can extend to a handlebody. Hence $\left|G_{o}\right| \leq A H_{g}$, and $A_{g}^{-} \leq A H_{g}^{-}$. Then $A_{g}^{-}=A H_{g}^{-}$.

By Proposition 4.74.10 and Remark 1.4 we finished the proof of Proposition 1.9. hence finished the proof of Theorem 1.3 .

\section{MaXimum orders of EXtendable aCtions on SuRfaces}

In this section we will determine the maximum orders of extendable cyclic and abelian group actions on orientable closed surfaces. For an extendable action on $\Sigma_{g}$, we will identify $\Sigma_{g}$ and its image $e\left(\Sigma_{g}\right)$ in $S^{3}$. Firstly we will prove two lemmas.

Lemma 5.1. Let $\langle h\rangle$ be a non-trivial cyclic group acting faithfully on $S^{3}$. $h$ preserves the orientation of $S^{3}$ and has a fixed point. Then the set of singular points in $S^{3} /\langle h\rangle$ is a $S^{1}$, with index $|\langle h\rangle|$, and $\pi_{1}\left(\left|S^{3} /\langle h\rangle\right|\right)$ is trivial.

Proof. Let $k$ be a factor of $|\langle h\rangle|$ and $k \neq|\langle h\rangle|$. By Lemma 3.8, the set of fixed points of $\left\langle h^{k}\right\rangle$ is a $S^{1}$. Hence it belongs to the set of fixed points of $\langle h\rangle$. Namely the set of singular points in $S^{3} /\langle h\rangle$ is a $S^{1}$, with index $|\langle h\rangle|$. Since $\pi_{1}\left(S^{3} /\langle h\rangle\right) \cong\langle h\rangle$, $\pi_{1}\left(\left|S^{3} /\langle h\rangle\right|\right)$ must be trivial. 
Lemma 5.2. Let $S_{H}^{3}$ be an integer homology 3-sphere, and $S_{g}$ be a genus $g \geq 0$ orientable closed subsurface in $S_{H}^{3}$. Then $S_{g}$ splits $S_{H}^{3}$ into two compact manifolds $M$ and $N$, each has the same integer homology groups as $V_{g}$.

Proof. Since $S_{H}^{3}$ is orientable, $S_{g}$ is two sided in $S_{H}^{3}$. If it does not split $S_{H}^{3}$, then there is a surjective homomorphism from $H_{1}\left(S_{H}^{3}\right)$ to $\mathbb{Z}$, which is a contradiction. Then we have the following MV-sequence:

$$
\begin{aligned}
& H_{3}\left(S_{H}^{3}\right) \longrightarrow H_{2}\left(S_{g}\right) \longrightarrow H_{2}(M) \oplus H_{2}(N) \longrightarrow H_{2}\left(S_{H}^{3}\right) \\
& H_{2}\left(S_{H}^{3}\right) \longrightarrow H_{1}\left(S_{g}\right) \longrightarrow H_{1}(M) \oplus H_{1}(N) \longrightarrow H_{1}\left(S_{H}^{3}\right)
\end{aligned}
$$

Clearly we have $H_{i}(M)=H_{i}(N)=0$ for $i>2$. By the first exact sequence, $H_{2}(M)=H_{2}(N)=0$. By the second exact sequence, $H_{1}(M) \oplus H_{1}(N) \cong \oplus_{i=1}^{2 g} \mathbb{Z}$. Then by the "half live half die" theorem, $H_{1}(M) \cong H_{1}(N) \cong \oplus_{i=1}^{g} \mathbb{Z}$.

\subsection{Maximum orders of cyclic extendable actions on surfaces.}

Proposition 5.3. $C E_{g}(-,+)=C_{g}^{-}$.

Proof. Clearly $C E_{g}(-,+) \leq C_{g}^{-}$. By (1) in Example2.2 and Example2.3, we have $C E_{g}(-,+)=C_{g}^{-}$.

Proposition 5.4. $C E_{g}(+,-)=2 g+2$.

Proof. Let $\langle h\rangle$ be a cyclic group acting on $\Sigma_{g}$. And the action is a type $(+,-)$ extendable action realizing the maximum order $C E_{g}(+,-)$. Then the action of $\left\langle h^{2}\right\rangle$ on $\Sigma_{g}$ can extend to some 3-manifold. Hence by Lemma 3.16, it can extend to a handlebody $V_{g}$. By (3) in Example 2.2, we have $\left|\left\langle h^{2}\right\rangle\right|>g-1$. Hence $V_{g} /\left\langle h^{2}\right\rangle$ is in the classes (A-D) in Lemma 4.1 .

Since $h$ reverses the orientation of $S^{3}$, it has a fixed point. Then $h^{2}$ has a fixed point. By Lemma 5.1, the set of singular points of $S^{3} /\left\langle h^{2}\right\rangle$ is a $S^{1}$, with index $\left|\left\langle h^{2}\right\rangle\right|$. Then $V_{g} /\left\langle h^{2}\right\rangle$ can only belong to classes (A-C). By Lemma 4.3. we have:

If $V_{g} /\left\langle h^{2}\right\rangle$ is in the class (A), then $\left|\left\langle h^{2}\right\rangle\right|=l=m=n=2$. Hence $|\langle h\rangle|=4$, and $g=2$.

If $V_{g} /\left\langle h^{2}\right\rangle$ is in the class (B), then $\left|\left\langle h^{2}\right\rangle\right|=m=n$. Hence $|\langle h\rangle|=2 n, g=n-1$.

If $V_{g} /\left\langle h^{2}\right\rangle$ is in the class (C), then $\left|\left\langle h^{2}\right\rangle\right|=n$. Hence $|\langle h\rangle|=2 n, g=n$.

Hence $|\langle h\rangle| \leq 2 g+2$. Then by (3) in Example 2.2 $C E_{g}(+,-)=2 g+2$.

Proposition 5.5. $C E_{g}(-,-)=2 g+1+(-1)^{g}$.

Proof. The proof is the same as above, except that if $V_{g} /\left\langle h^{2}\right\rangle$ is in the class (B).

In this case, $\Sigma_{g} /\left\langle h^{2}\right\rangle$ is a $S^{2}$ with four singular points. Let $\Theta$ be the set of singular points in $S^{3} /\left\langle h^{2}\right\rangle$. By Lemma 5.1. $\Theta$ is a $S^{1}$, and $S^{3} /\left\langle h^{2}\right\rangle$ is an integer homology 3-sphere. By Lemma [5.2 $\left|\Sigma_{g} /\left\langle h^{2}\right\rangle\right|$ splits $\left|S^{3} /\left\langle h^{2}\right\rangle\right|$ into two compact manifolds $M$ and $N$. Let $\bar{h}$ be the image of $h$ in $\langle h\rangle /\left\langle h^{2}\right\rangle$, and $N(\Theta)$ be a $\bar{h}$-invariant regular neighbourhood of $\Theta$. Then $\bar{h}$ acts on $\overline{M-N(\Theta)}$. By Lemma 5.2, $\overline{M-N(\Theta)}$ will have the same homology groups as $V_{2}$. Then by Lemma 4.5, $\bar{h}$ has a fixed point in $\overline{M-N(\Theta)}$. By Lemma 4.4, we have that $\left|\left\langle h^{2}\right\rangle\right|$ is odd. Hence $g$ is even. Namely we have $C E_{g}(-,-) \leq 2 g+1+(-1)^{g}$.

Then by $(2)$ and $\left(2^{\prime}\right)$ in Example 2.2, $C E_{g}(-,-)=2 g+1+(-1)^{g}$.

Proposition 5.6. The type (Mix) extendable cyclic group action does not exist.

Proof. For a type (Mix) extendable $G$-action, there is a surjective homeomorphism $G \rightarrow \mathbb{Z}_{2} \oplus \mathbb{Z}_{2}$. Hence $G$ can not be cyclic. 


\subsection{Maximum orders of abelian extendable actions on surfaces.}

Proposition 5.7. $A E_{g}(+,-)=A E_{g}(-,+)=A E_{g}(-,-)=2 A E_{g}$.

Proof. Let $\mathcal{T}$ be one of $(+,-),(-,+)$ and $(-,-)$. Clearly $A E_{g} \mathcal{T} \leq 2 A E_{g}$. Then by (4), (5) and (6) in Example 2.2, we have $A E_{g} \mathcal{T}=2 A E_{g}$.

Proposition 5.8. For odd $g>1$, the type (Mix) extendable abelian group action does not exist.

Proof. Suppose there is a type (Mix) extendable abelian $G$-action on $\Sigma_{g}$. Then there exist $h_{1}, h_{2} \in G$ such that $h_{1}$ preserves the orientation of $\Sigma_{g}$ and reverses the orientation of $S^{3}$, and $h_{2} \in G$ reverses the orientation of $\Sigma_{g}$ and preserves the orientation of $S^{3}$. Then both $h_{1}$ and $h_{2}$ change the two sides of $\Sigma_{g}$.

By Lemma 5.2, $\Sigma_{g}$ splits $S^{3}$ into $M$ and $N$, and $H_{1}(M) \simeq H_{1}(N) \simeq \oplus_{i=1}^{g} \mathbb{Z}$. Let $i_{M}: \Sigma_{g} \rightarrow M, i_{N}: \Sigma_{g} \rightarrow N$ be the inclusions. We can choose a basis of $H_{1}\left(\Sigma_{g}\right)$, denoted by $\left\{\alpha_{1}, \alpha_{2}, \cdots, \alpha_{g}, \beta_{1}, \beta_{2}, \cdots, \beta_{g}\right\}$, satisfying the following conditions:

(1) $\left\{i_{M_{*}}\left(\alpha_{k}\right)\right\}$ is a basis of $H_{1}(M), k=1,2, \cdots, g$.

(2) $\left\{i_{N *}\left(\beta_{k}\right)\right\}$ is a basis of $H_{1}(N), k=1,2, \cdots, g$.

(3) $i_{N *}\left(\alpha_{k}\right)=0$ and $i_{M *}\left(\beta_{k}\right)=0, k=1,2, \cdots, g$.

Then consider the intersection product of $H_{1}\left(\Sigma_{g}\right)$. By the condition (3) we have $\alpha_{i} \bullet \alpha_{j}=0$ and $\beta_{i} \bullet \beta_{j}=0,1 \leq i, j \leq g$, hence we have:

$$
\left(\begin{array}{c}
\alpha_{1} \\
\vdots \\
\alpha_{g} \\
\beta_{1} \\
\vdots \\
\beta_{g}
\end{array}\right) \bullet\left(\alpha_{1}, \cdots, \alpha_{g}, \beta_{1}, \cdots, \beta_{g}\right)=\left(\begin{array}{cc}
0 & X \\
-X^{t} & 0
\end{array}\right)
$$

Here $X=\left(x_{i, j}\right)$ is a $g \times g$ matrix, and $x_{i, j}=\alpha_{i} \bullet \beta_{j} . X^{t}$ is the transposition of $X$. There are $g \times g$ matrices $A, B, C, D$, such that:

$$
\begin{aligned}
& h_{1 *}\left(\alpha_{1}, \cdots, \alpha_{g}, \beta_{1}, \cdots, \beta_{g}\right)=\left(\alpha_{1}, \cdots, \alpha_{g}, \beta_{1}, \cdots, \beta_{g}\right)\left(\begin{array}{cc}
0 & A \\
B & 0
\end{array}\right) \\
& h_{2 *}\left(\alpha_{1}, \cdots, \alpha_{g}, \beta_{1}, \cdots, \beta_{g}\right)=\left(\alpha_{1}, \cdots, \alpha_{g}, \beta_{1}, \cdots, \beta_{g}\right)\left(\begin{array}{cc}
0 & C \\
D & 0
\end{array}\right)
\end{aligned}
$$

By computing the intersection product, we have the following:

$$
\begin{aligned}
& \left(\begin{array}{cc}
0 & B^{t} \\
A^{t} & 0
\end{array}\right)\left(\begin{array}{cc}
0 & X \\
-X^{t} & 0
\end{array}\right)\left(\begin{array}{cc}
0 & A \\
B & 0
\end{array}\right)=\left(\begin{array}{cc}
0 & X \\
-X^{t} & 0
\end{array}\right) \\
& \left(\begin{array}{cc}
0 & D^{t} \\
C^{t} & 0
\end{array}\right)\left(\begin{array}{cc}
0 & X \\
-X^{t} & 0
\end{array}\right)\left(\begin{array}{cc}
0 & C \\
D & 0
\end{array}\right)=\left(\begin{array}{cc}
0 & -X \\
X^{t} & 0
\end{array}\right)
\end{aligned}
$$

Hence we have $A^{t} X B=-X^{t}$ and $C^{t} X D=X^{t}$. Since the group $G$ is abelian, $h_{1} h_{2}=h_{2} h_{1}$. Then we have:

$$
\left(\begin{array}{cc}
0 & A \\
B & 0
\end{array}\right)\left(\begin{array}{cc}
0 & C \\
D & 0
\end{array}\right)=\left(\begin{array}{cc}
0 & C \\
D & 0
\end{array}\right)\left(\begin{array}{cc}
0 & A \\
B & 0
\end{array}\right)
$$

This is equivalent to $A D=C B$ and $B C=D A$.

The $g \times g$ matrices $A, B, C, D$ and $X$ are all non-degenerate. By computing the determinants we have $|A||X||B|=(-1)^{g}|X|,|C||X||D|=|X|$ and $|A||D|=|C||B|$. Hence $(-1)^{g}=|A B C D|=|A D|^{2}$, and $g$ is even. 
Proposition 5.9. For even $g>1, A E_{g}(M i x)=2 g+4$.

Proof. Let $G$ be an abelian group acting on $\Sigma_{g}$. The action is a type (Mix) extendable action, and $|G|=A E_{g}(M i x)$. Let $G_{o}$ be the subgroup of $G$ containing all elements preserving both the orientations of $\Sigma_{g}$ and $S^{3}$, then $|G|=4\left|G_{o}\right|$.

Choose $h \in G$ such that it reverses the orientation of $S^{3}$. Let Fix $(h)$ be the set of fixed points of $h$. By Lemma 3.8, Fix $(h)$ is a $S^{0}$ or $S^{2}$. Since $G$ is abelian, every element of $G$ keeps Fix $(h)$ invariant. Let $G_{o}^{\prime}$ be the subgroup of $G$ containing all elements preserving both the orientations of Fix $(h)$ and $S^{3}$. If $F i x(h) \cong\{0,1\}$, then preserving the orientation of Fix $(h)$ means fixing 0 and 1.

Case 1: $G_{o}^{\prime} ¥ \mathbb{Z}_{2} \oplus \mathbb{Z}_{2}$ and $\left|G_{o}^{\prime}\right|>2$.

Then $G_{o}^{\prime}$ is a cyclic group of order $\left|G_{o}^{\prime}\right| \geq 3$, and the $G_{o}^{\prime}$-action on $S^{3}$ has a fixed point. By Lemma 5.1, the set of singular points of $S^{3} / G_{o}^{\prime}$ is a circle $\Theta$, with index $\left|G_{o}^{\prime}\right|$, and $\pi_{1}\left(\left|S^{3} / G_{o}^{\prime}\right|\right)$ is trivial. $\left|S^{3} / G_{o}^{\prime}\right|$ is an integer homology 3-sphere.

Let $\Theta^{\prime}$ be the pre-image of $\Theta$ in $S^{3}$, then $\Theta^{\prime}$ is also a circle. Since $\left|G_{o}^{\prime}\right| \geq 3, \Sigma_{g}$ intersects $\Theta^{\prime}$ transversely. Hence $\left|\Sigma_{g} / G_{o}^{\prime}\right|$ is an orientable closed surface in $\left|S^{3} / G_{o}^{\prime}\right|$. Then $\Sigma_{g} / G_{o}^{\prime}$ is an orientable 2-suborbifold in $S^{3} / G_{o}^{\prime}$, namely $G_{o}^{\prime}$ preserves both the orientations of $\Sigma_{g}$ and $S^{3}$. Since $\left|G / G_{o}^{\prime}\right| \leq 4$, we have $G_{o}^{\prime}=G_{o}$. By Lemma 5.2 $\left|\Sigma_{g} / G_{o}\right|$ splits $\left|S^{3} / G_{o}\right|$ into two compact manifolds $M$ and $N$.

Let $g^{\prime}$ be the genus of $\left|\Sigma_{g} / G_{o}\right|$, and $k$ be the number of singular points in $\Sigma_{g} / G_{o}$. Then $k$ must be even. By the Riemann-Hurwitz formula, we have:

$$
2-2 g=\left|G_{o}\right|\left(2-2 g^{\prime}-k\left(1-\frac{1}{\left|G_{o}\right|}\right)\right) .
$$

By Example 2.4, $\left|G_{o}\right| \geq(2 g+4) / 4=g / 2+1$. Then we have:

$$
\left(g^{\prime}, k,\left|G_{o}\right|\right)=(2,0, g-1),(1,2, g),(0,4, g+1),(0,6, g / 2+1) .
$$

Notice that there is a $G / G_{o} \cong \mathbb{Z}_{2} \oplus \mathbb{Z}_{2}$-action on $\left(\Sigma_{g} / G_{o}, S^{3} / G_{o}\right)$. It satisfies a similar condition of the type (Mix) extendable action.

If $\left(g^{\prime}, k,\left|G_{o}\right|\right)=(2,0, g-1)$, then $\Theta$ is contained in one side of $\left|\Sigma_{g} / G_{o}\right|$ in $\left|S^{3} / G_{o}\right|$. Hence the required $\mathbb{Z}_{2} \oplus \mathbb{Z}_{2}$-action does not exist.

If $\left(g^{\prime}, k,\left|G_{o}\right|\right)=(1,2, g)$, then $\left|\Sigma_{g} / G_{o}\right| \simeq T^{2}$. In the proof of Proposition 5.8, we only used homology theories, and the proof does not depend on $g>1$. Hence by a similar proof, the required $\mathbb{Z}_{2} \oplus \mathbb{Z}_{2}$-action on $\left(\left|\Sigma_{g} / G_{o}\right|,\left|S^{3} / G_{o}\right|\right)$ does not exist.

If $\left(g^{\prime}, k,\left|G_{o}\right|\right)=(0,4, g+1)$, then $\left|\Sigma_{g} / G_{o}\right| \simeq S^{2}$ and $k=4$. Suppose $\left|\Sigma_{g} / G_{o}\right|$ intersects $\Theta$ at $\{A, B, C, D\}$, see Figure 5 .

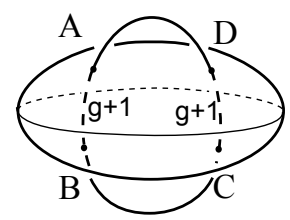

Figure 5. $\Sigma_{g} / G_{o}$ and $\Theta$ in $S^{3} / G_{o}$

If the required $\mathbb{Z}_{2} \oplus \mathbb{Z}_{2}$-action exists, then there exists $\eta \in \mathbb{Z}_{2} \oplus \mathbb{Z}_{2}$, it preserves the orientation of $S^{3} / G_{o}$ and reverses the orientation of $\Sigma_{g} / G_{o}$. Then on $\{A, B, C, D\}$ it has no fixed point. Otherwise, in $S^{3} / G$ the image of the fixed point will have non-abelian local group. Since $\eta$ changes the two sides of $S^{3} / G_{o}$, we can assume $\eta(A B)=A D$. Then $\eta$ will have order 4 , which is a contradiction. 
Hence we have $\left(g^{\prime}, k,\left|G_{o}\right|\right)=(0,6, g / 2+1)$, and $|G|=2 g+4$.

Case 2: $G_{o}^{\prime} \cong \mathbb{Z}_{2} \oplus \mathbb{Z}_{2}$ or $\left|G_{o}^{\prime}\right|=2$.

If $|G|=A E_{g}($ Mix $)>2 g+4$, then $|G|>8$. Hence $\left|G_{o}^{\prime}\right| \neq 2$. Then $G_{o}^{\prime} \cong \mathbb{Z}_{2} \oplus \mathbb{Z}_{2}$, and $|G|=16$. By Proposition 5.8 and $1.9, g$ can only be 4 .

Let $G^{\prime}$ be the subgroup of $G$ containing all elements preserving the orientation of $\Sigma_{4}$. By the proof of Proposition 4.10, the $G^{\prime}$-action can extend to $V_{4}$. Since $\left|G / G_{o}^{\prime}\right| \leq 4$ and $|G|=16$, we have $G / G_{o}^{\prime} \cong \mathbb{Z}_{2} \oplus \mathbb{Z}_{2}$. Then $G^{\prime} \cong \mathbb{Z}_{2} \oplus \mathbb{Z}_{2} \oplus \mathbb{Z}_{2}$ or $\mathbb{Z}_{4} \oplus \mathbb{Z}_{2}$. Let $\mathcal{G}$ be the induced finite graph of finite groups of $V_{4} / G^{\prime}$. On one hand $\chi(\mathcal{G})=(1-4) / 8=-3 / 8$. On the other hand, vertices and edges of $\mathcal{G}$ have orders 1,2 or 4 . Hence $4 \chi(\mathcal{G}) \in \mathbb{Z}$. This is a contradiction.

By Proposition 5.35 .9 and Remark 1.4 we finished the proof of Proposition 1.8 hence finished the proof of Theorem 1.2 .

\section{REFERENCES}

[1] M. Boileau, S. Maillot, J. Porti, Three-dimensional orbifolds and their geometric structures, Panoramas et Synthèses 15. Société Mathématique de France, Paris, 2003.

[2] M. Boileau, J. Porti, Geometrization of 3-orbifolds of cyclic type, Astérisque No. 272 (2001).

[3] S. Eilenberg, Topologie du plan, Fundamenta Mathematicae, vol. 26 (1936), 61-112.

[4] W. J. Harvey, Cyclic groups of automorphisms of a compact Riemann surface, Quart. J. Math. Oxford Ser. (2) 17 (1966), 86-97.

[5] G. Livesay, G. R. Involutions with two fixed points on the three-sphere, Ann. of Math. (2) 78 (1963) 582-593.

[6] C. Maclachlan, A bound for the number of automorphisms of a compact Riemann surface, J. London Math. Soc. 44 (1969), 265-272.

[7] D. McCullough, A. Miller, B. Zimmermann, Group actions on handlebodies, Proc. London Math. Soc. (3) 59, (1989), 373-416.

[8] M. Reni, B. Zimmermann, Handlebody orbifolds and Schottky uniformizations of hyperbolic 2-orbifolds, Proc. Amer. Math. Soc. 123 (1995), 3907-3914.

[9] M. Reni, B. Zimmermann, Extending finite group actions from surfaces to handlebodies, Proc. Am. Math. Soc. 124 (1996), 2877-2887.

[10] P. A. Smith, New results and old problems in finite transformation groups, Bull. AMS, 66 (1960), 401-415.

[11] F. Steiger, maximalen Ordnungen periodischer topologischer Abbildungen geschlossener Flachen in sich, Comment. Math. Helv. 8 (1935), 48-69.

[12] W. P. Thurston, The geometry and topology of three-manifolds, Lecture notes, 1978.

[13] S. C. Wang, Maximum orders of periodic maps on closed surfaces, Topology Appl. 41 (1991), 255-262.

[14] C. Wang, S. C. Wang, Y. M. Zhang, B. Zimmerman, Extending finite group actions on surfaces over $S^{3}$, Topology Appl. 160 (2013), 2088-2103.

[15] B. Zimmermann, Genus actions of finite groups on 3-manifolds, Michigan Math. J. 43 (1996), 593-610.

School of Mathematical Sciences, University of Science and Technology of China, HeFEI 230026, CHINA

E-mail address: chao_wang_1987@126.com

Mathematics School, Jilin University, Changchun 130012, ChinA

E-mail address: zym534685421@126.com 